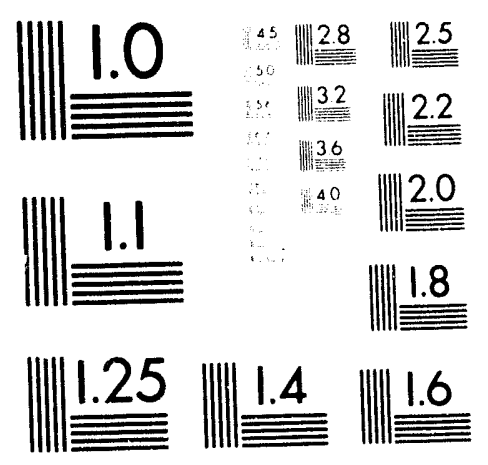



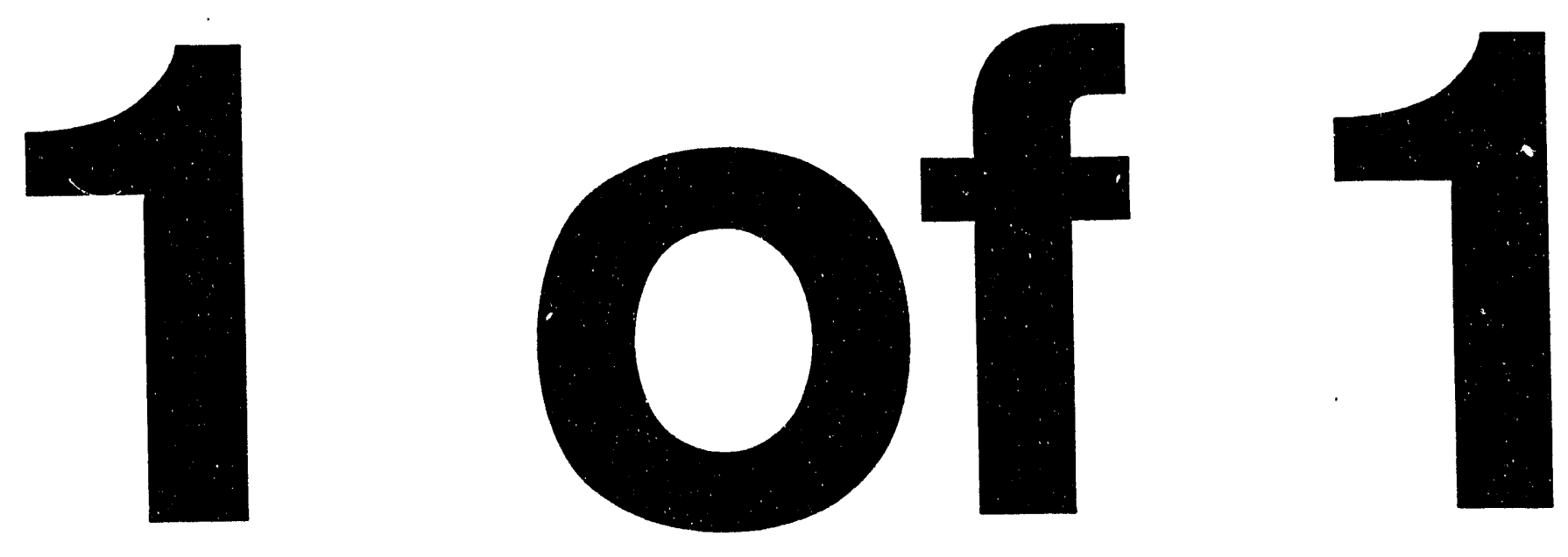


$$
\begin{aligned}
\text { Cony.. } 9305 i 1-415 & \text { SLAC-PUB-6108 } \\
& \text { SLAC/SSRL-0022 } \\
& \text { MaY } 1993 \\
& \text { SSRL-ACD }
\end{aligned}
$$

\title{
Photon Pulse Filtering and Modulation Based on the Extreme Temporal Compression and Correlated Energy Spread of the Electron Bunches in the SLAC Linac Coherent Light Source (LCLS)*
}

\author{
R. Tatchyn \\ Stanford Linear Accelerator Center, Stanford, CA 94305, USA
}

\section{Abstract}

The LCLS photon pulses are expected to attain unprecedented levels of brightness and brevity in the 300 $400 \mathrm{eV}$ range. Nominally, the photon pulse length will be dominated by the electron bunch length, while the performance of conventional $x$-ray reflecting and bandshaping optics will be limited by : 1) peak power damage, and 2) transform-limited monochromatization. In this paper we describe how: 1) the correlated energy spread in the electron bunch can be used to selectably compress the LCLS photon pulses to below their nominal length; 2) gas optics can be used to mitigate peak damage problems; 3) the LCLS pulse structure can, in principle, accommodate schemes based on "disposable" optics; and 4) pulse lengthening schemes can be used to extend the attainable degree of mon inhomatization.

\section{INTRODUCTION}

A list of parameter values characterizing the coherent output photon pulses of the LCLS -perated at $E=7 \mathrm{GeV}$ is given in Table $1[1,2]$. Based on current 3-dimensional models and analyses of the Self Amplified Spontaneous Emission (SASE) process [3] in a Free-Electron Laser, the photon bunch length is associated with the physical length

Table 1

LCLS Photon Output Parameters

\begin{tabular}{|lr|}
\hline 1st Harmonic Wavelength $(\lambda)$ & $40 \AA-30 \AA$ \\
\hline Peak Power $\left(\mathrm{P}_{\text {peak }}\right)$ & $10 \mathrm{GW}$ \\
\hline Beam Diam. $\left(D_{\mathrm{W}}\right)$ at Optics Locations & $1 \mathrm{~mm}-2 \mathrm{~mm}$ \\
\hline Full Pulse Duration $\left(\sqrt{2 \pi \sigma_{\tau}}\right)$ & $0.5 \mathrm{ps}$ \\
\hline Full Pulse Length & $150 \mu$ \\
\hline Pulse Repetition Rate & $120 \mathrm{~Hz}$ \\
\hline Energy per Pulse & $-5 \mathrm{~mJ}$ \\
\hline Peak Power Density (Norm. Incidence) & $\sim 10^{16} \mathrm{~W} / \mathrm{m}^{2}$ \\
\hline
\end{tabular}

of an ultrashort electron bunch containing $0.5 \times 10^{10}-10^{11}$ electrons $(1-2 n C)$. Due to the sensitivity of the FEI gain process to the local particle density in the bunch, however, it should be noted that further refinements in SASE models (or actual experimental measurements) may demonstrate a narrowing of the photon pulses to values significantly

\footnotetext{
* Supported by DOE Offices of Basic Energy Sciences and High Energy and Nuclear Physics and Department of Energy Contract DEAC.03-76SF0015.

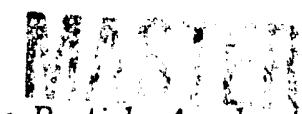

Presented at the Particle Accelerntor Conference (PAC 93), Woshington, DC: May 17-20. 1993 smaller than those listed in the table.

An important property of the electron bunch accelerated through the linac is the development of an energy gradient (or "correlated energy spread") in the forward direction due to electrons in the front of the bunch loading the accelerating fields addressed by the trailing particles [4]. This energy spread is superimposed on the "uncorrelated" energy spread of the bunch characterized by its (stochastic) emittance parameters. For our purposes, we note that these energy spreads show up as, respectively, "inhomogeneous" and "homogenequs" line broadenings in the FEL photon pulse. Due to the $\gamma^{2}$ (or $E^{2}$ ) dependence of the FEL's output photon energy, these broadenings can be shown to appear with roughly twice the relative size in the photon spectrum as in the electron bunch energy.

In a recent workshop [5] on selected scientific applications of the LCLS, attention was focussed on the unprecedented spectral and temporal parameter ranges of the LCLS for opening up new experimental possibilities. In this paper we review selected approaches for utilizing the cited and tabulated properties of the LCLS in conjunction with specialized techniques and instrumentation [6] to further extend the output parameter ranges and potential uses of this novel $\mathrm{x}$-ray source.

\section{LCLS PULSE COMPRESSION TECHNIQUES}

Perhaps the most direct and effective approach to photon pulse compression would be to reduce the length of the linac electron bunches even further. As described elsewhere [7], electron bunch compression in the SLAC linac is already necessary for attaining the pulse lengths given in Table 1. Preliminary technical assessments of further applications of this technique indicate that another factor of 10 (viz., down to $50 \mathrm{fs}$ ) could perhaps be feasible [8]. This method, while non-trivial to implement, would evidently be the most attractive, since increasing the particle density will tend to amplify the FEL gain, leading to increased numbers of in-band photons. A less efficient, alternative method, based on utilizing the inhomogeneous energy gradient of the photon be $\mathrm{m}$ in the forward di ection, could also be used to extract $x$-ray pulses from the LCLS with durations down to 50 fs and beyond. The procedure for accomplishing this, based on normal-incidence dispersion by a transmission grating with a chirped period a, is schematized at the top of Fig. 1. If we assume that the inhomogeneous energy spread in the photon pulse can be resolved into two levels, each associated with a distinct longitudinal region, it is apparent that the photons from the 
lower-energy region will be dispersed into a larger angle than the ones from the forward region. As the dispersed photons "scan" away from the $\mathrm{z}$ axis, a suitably-sized aperture will segregate out a temporally -resolved subinterval of the initial pulse. Since the photons are extracted in a volume which is significantly truncated in both the $x$ and $z$ directions, and since the indicated diffraction will incur a $90 \%-95 \%$ loss into 1 st order, it is evident that significant loss factors will be associated with the implementation of this technique. In Table

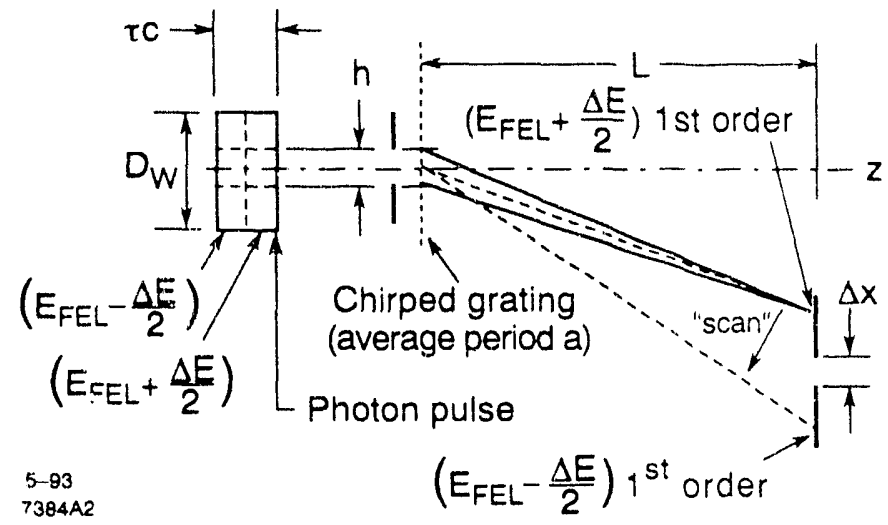

Figure 1. Temporal pulse compression based on chirped photon energy in the source pulse.

2 we show some of the attainable pulse lengths $\left(\tau^{\prime}\right)$, correlated and inhomogeneous energy spreads $\Delta E_{i n h}$ and $\Delta E_{\text {corr }}$, grating line densities $(1 / \mathrm{m})$, exposed lines $N$, and expected loss factors associated with compressing an 0.5 ps LCLS pulse.

Table 2

$\mathrm{D}_{\mathrm{W}}=0.001 \mathrm{~m} ; \mathrm{L}=2 \mathrm{~m} ; \Delta \mathrm{EFEL}_{\mathrm{EFEL}}=0.001$

\begin{tabular}{|c|l|l|l|l|l|l|l|}
\hline$\tau$ & $\mathrm{h}[\mathrm{m}]$ & $\mathrm{N}$ & $\mathrm{l} / \mathrm{m}$ & $\frac{\Delta E_{\text {inh }}}{\Delta E_{\text {FEL }}}$ & $\frac{\Delta E_{\text {corr }}}{E_{\text {LINAC }}}$ & $\begin{array}{l}\mathrm{\tau}^{\prime}[\mathrm{fs}] \\
\times 10^{1}\end{array}$ & $\begin{array}{l}\text { loss } \\
\text { factor } \\
\times 10^{-3}\end{array}$ \\
\hline 0.5 & 25 & 10 & 0.4 & 2 & 0.001 & 25 & 125 \\
\hline 0.5 & 10 & 25 & 2.5 & 5 & 0.0025 & 10 & 20 \\
\hline 0.5 & 5 & 50 & 10 & 10 & 0.005 & 5 & 5 \\
\hline 0.5 & 2.5 & 100 & $4 \mathrm{C}$ & 20 & 0.01 & 2.5 & 1.25 \\
\hline 0.5 & 1.25 & 200 & 160 & 40 & 0.02 & 1.25 & 0.31 \\
\hline
\end{tabular}

\section{PEAK INTENSITY AND MATERIALS DAMAGE ISSUES}

At normal incidence, it is easy to estimate from typical attenuation coefficients in the soft $x$-ray range $[9]$ and Table 1 that $1 \mathrm{eV}$ or more of energy can be deposited per atom in 0.5 ps. Keeping in mind that typical atomic or molecular lattice binding energies are of the order of $1 \mathrm{eV}$ and considering the relative time constants and efficiencies of primary energy-removal channels, it can be argued [ $\sigma]$ that the probability of fragmentation must start becoming appreciable under the cited conditions. We can consequently expect enhanced probability of damage in solid state materials by the LCLS photons from two primary effects: 1) lattice disruption/ablation, and 2) photoemissively-generated field stresses.

An intuitively evident way to mitigate peak intensity damage is to dilute the energy deposited per unit area (and thereby per atom) by the artifice of grazing incidence. As described in a related paper [2], this approach has been used to generate a practical design for a mirror station for the LCLS. Nevertheless, future $x$-ray FEL configurations can be posited (e.g., the tapered wiggler) whose peak outputs may be too high to be adequately handled by solid-state specular reflection even at extreme grazing incidence. To this end, we consider two alternative options: 1) gas optics, and 2) disposable normal-incidence optics.

Two possible schemes utilizing gas optics are shown in Fig. 2. The first, a single-jet "gas prism" deflects the

\section{Gas Optics}
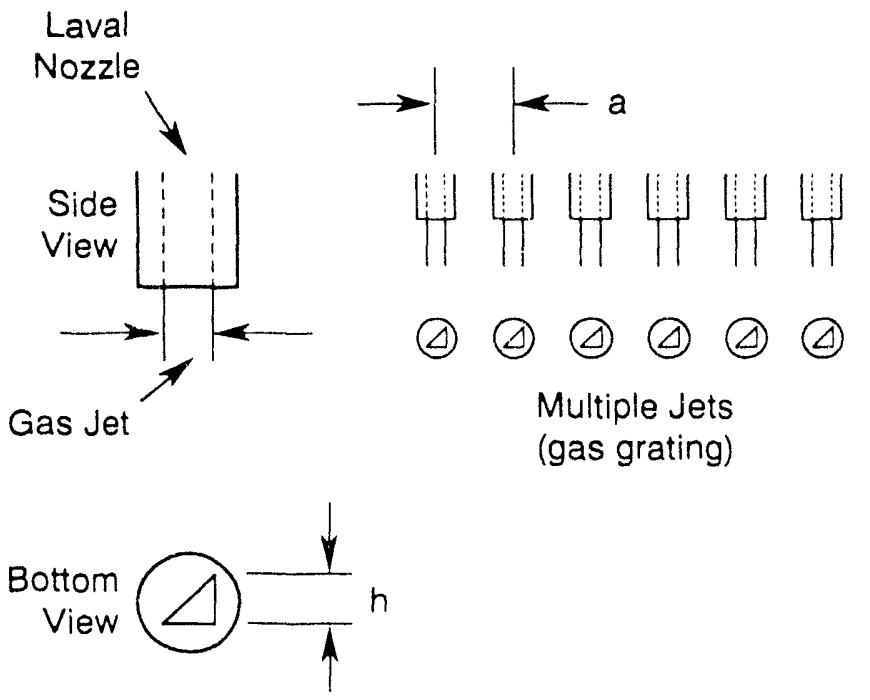

Single Jet (prism)

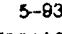

$7384 A 6$

Figure 2. Gas jet configurations for x-ray deflection.

LCLS output beam by inducing a phase gradient in its wavefront, similarly to an ordinary optical prism. The major difference lies in the rather large differential attenuation accompanying the phase gradient, which can incur an appreciable intensity-loss penalty per degree of deflection. The second configuration is a multi-jel "gas grating" with period a, enabling a minimum deflection angle of $\lambda / a$ to be attained. Here, however, the relative efficiency of the diffracted orders will be determined by the density contrast attained in the gas and the absolute efficiency by the average gas thickness, and it will be a difficult task to generate thin gas sheets with significant density or particle-number contrasts for grating periods extending down $1010 \mu$ and 
beyond. In either configuration, numerical studies indicate relatively lossy and weak steering using low $-Z$ neutral gases, including $\mathrm{H}_{2}$. Although fully or partially ionized plasmas could offer more advantageous riptical constants for effective beam steering, the preparation of sufficiently small and dense jets would in general be expected to be more difficult than with non-ionized gases.

Given these observations, we note that the relatively sparse pulse structure $(120 \mathrm{~Hz})$ of the LCLS can easily allow mechanical motion of optical surfaces and shutters over distances significantly greater than the beam waist to occur between pulses. This makes feasible the notion of using "disposable" optics at normal or near-normal incidence to deflect or otherwise process the LCLS beam. For example, with a beam spot size of $1 \mathrm{~mm}$, we can estimate that if the 1 $\mathrm{mm}$ area of impact is totally obliterated by one pulse, we would require a renewal rate of the optical surface of about 1 $\mathrm{m}^{2}$ /hour. For ultra-thin optics (e.g., zone plates or transmission gratings), suitably-placed rotating shutters could be used to effectivaly trap and collect the debris, and recovery and re-fabrication schemes could perhaps bo developed to sustain economic feasibility.

\section{PULSE-LENGTH LIMITATIONS ON COHERENCE}

Under the condition that the correlated and uncorrelated energy spreads in the electron bunch are equal, the FEL. radiation spectrum will be broadened to more than twice its natural relative width. For larger correlated energy spreads the inhomogeneous broadening will be correspondingly greater. The present lack of detailed knowledge of the actual spread in the width of the LCLS pulses underscores the desirability of being able to monochromatize the emitted FEL photon pulses, and a number of important scientific applications such as, e.g., high-precision absorption or photoemission spectroscopy, will demand it.

Referring to Fig. 3, we note that conventional soft $x$-ray

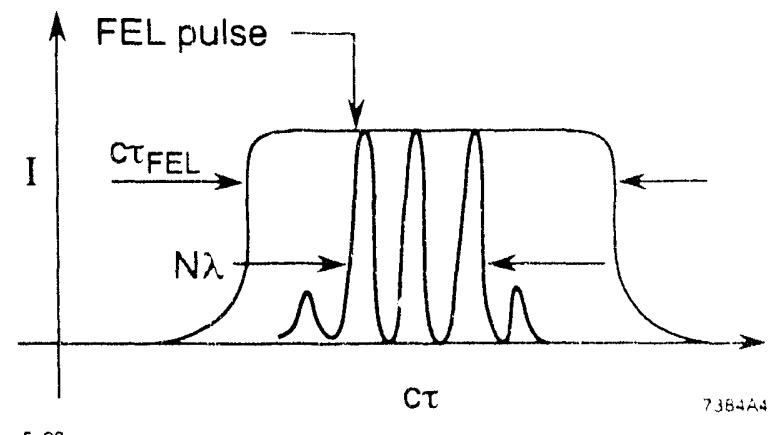

Figire 3. Total vs, coherence length of L.CLS pulse.

monochromators usually process temporal pulses whose physical length $(\tau c)$ is considerably longer than the coherence length $(\mathrm{N} \lambda)$ of the wave trains corresponding to their resolving power. For the LCLS, however, the pulse length could approach or even become smaller than this coherence length. For a monochromator with maximum resolving power $R_{M}$, operating at normal incidence dispersion, the actual pulse-length-limited resolving power $R$ can be expressed as

$$
R \simeq\left[\left(\frac{1}{R_{M}}\right)^{2}+\left(\frac{\lambda^{\prime}}{\tau}\right)^{2}\right]^{-0.5}
$$

where $\lambda^{\prime}=\lambda+\left(D_{w^{a}} a / 2 L\right), \mathrm{D}_{w}$ is the beam diameter, a is the grating period, and $L$ is the dispersion length. Since variation of the monochromatort parameters can at best be used to reduce $\lambda^{\prime}$ to $\lambda$, it is evident that the most effective way to maintain $\mathrm{R} \rightarrow \mathrm{R}_{\mathrm{M}}$ is to lengthen the LCLS pulse. One way to accomplish this is to utilize the diffraction process itself, which lengthens the pulse diffracted into any order. By selecting a small fraction of the dilated pulse with suitable spatial and angular filtering, one can obtain an attenuated and dilated pulse suitable for further monochromatization. An alternative approach, which would also reduce the number of in-band photons (by reducing gain), would be to underutilize the compression stages in the linac to produce longer electron bunch lengths with smaller peak currents.

\section{REFERENCES}

[1] H. Winick, K. Bane, R. Boyce, K. Halbach, K.-J. Kim, G. Loew, P. Morton, H.-D. Nuhn, J. Paterson, C. Pellegrini, P. Pianetta, D. Prosnitz, J. Rosenzweig, J. Seeman, T. Raubenheimer, T. Scharlemann, R. Tatchyn, G. Travish, V. Vylet, M.Xie, "A 2-4 nm Linac Coherent Light Source (LCLS) Using the SLAC Linac," this conference F7, 1993.

[2] R. Tatchyn and P. Pianetta, "X-Ray Beam Lines and Beam Line Components for the SLAC Linac Coherent Light Source (LCLS)," this conference - Mb29, 1993.

[3] K. Kim, M. Xie, E. Scharlemann, C. Pellegrini, G. Travish, "Performance Characteristics, Optimization, and Error Tolerances of a $4-n m$ FEL Based on the SLAC. Linac," this conference - Mb26, 1993.

[4] R. B. Palmer, "Prospects for High Energy e+e-Linear Colliders," Annu. Rev. Nucl. Part. Sci. 1990.40:529-92.

[5] W. Spicer, J. Arthur, H. Winick, eds., Proceedings of the Workshop on Scientific Applications of Short Wavelength Coherent Light Sources, Stanford, CA, October 21, 1992, SLAC-Report-414, February 1993.

[6] R. Tatchyn, "LCLS Optics: Selected Technological Issues and Scientific Opprtunities, " ibid., pp. 93-119.

[7] J. Seeman, K. Bane, T. Raubenheimer, "Electron Transport of a Linac Coherent Light Source (LCLS) Using the SLAC Linac," this conference - Jb19, 1993.

[8] T. Raubenheimer and K. Bane, private communication.

[9] B. L. Henke, P. !.ee, T. J. Tanaka, R. L. Shimabukuro, and B. K. Fujikawa, "The Atomic Scattering Factor, fl + if2, for 44 Elements and for the 100 to $2000 \mathrm{eV}$ Photon Energy Region," AIP Conference Proceedings No. 75 , $340(1982)$. 

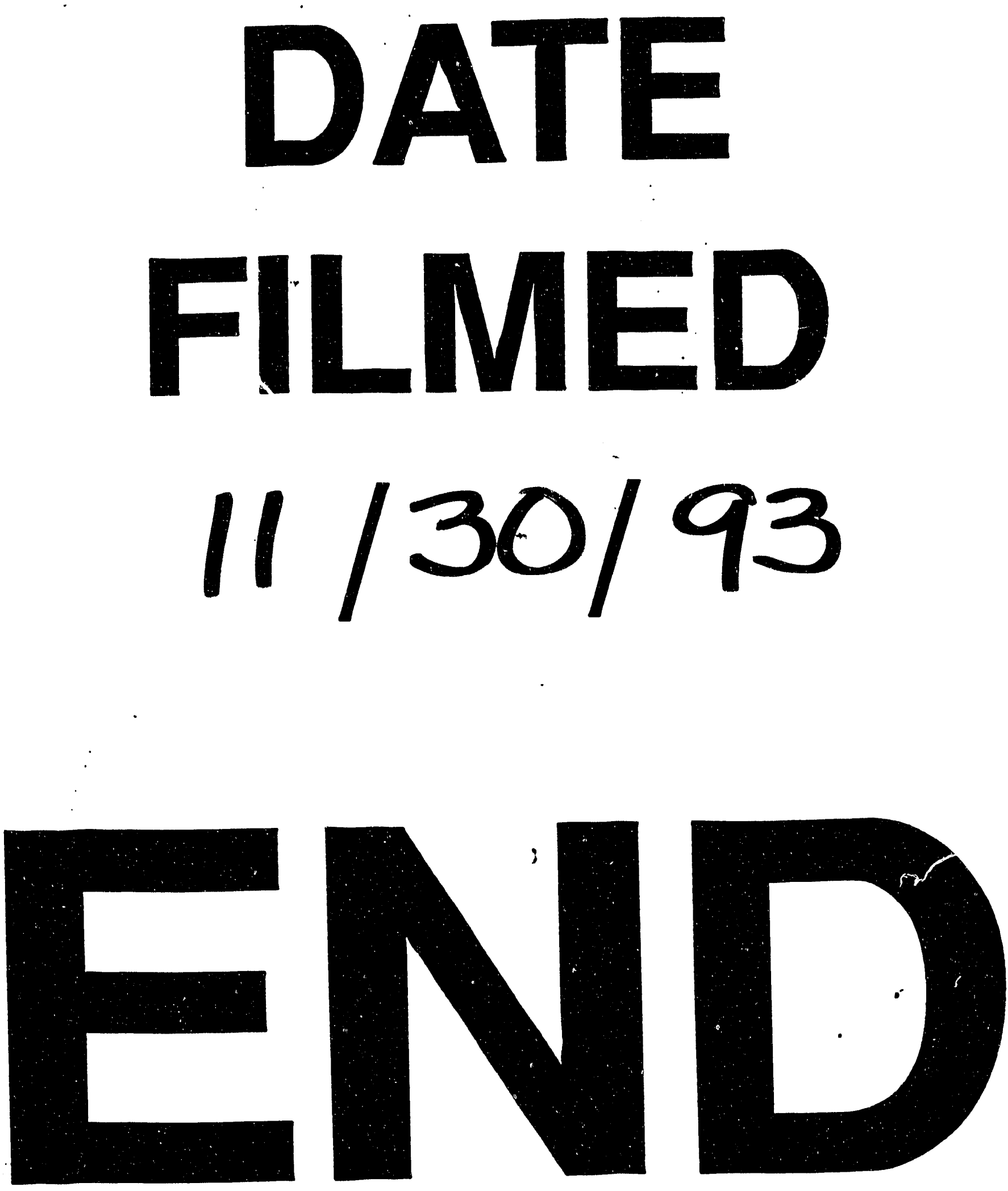
